\title{
Image mixed gaussian and impulse noise elimination based on sparse representation model
}

\author{
Ahmed Abdulqader Hussein ${ }^{1}$, Sabahaldin A. Hussain ${ }^{2}$, Ahmed Hameed Reja ${ }^{3}$ \\ ${ }^{1,3}$ Department of Electromechanical Engineering, University of Technology, Baghdad, Iraq \\ ${ }^{2}$ Computer Science Department, College of Science, Aljufra University, Libya
}

\section{Article Info \\ Article history: \\ Received Dec 7, 2020 \\ Revised Jul 28, 2021 \\ Accepted Aug 4, 2021}

\section{Keywords:}

Gaussian noise

Image denoising

Impulse noise

Mixed noise

Sparse representation model

\begin{abstract}
A modified mixed Gaussian plus impulse image denoising algorithm based on weighted encoding with image sparsity and nonlocal self-similarity priors regularization is proposed in this paper. The encoding weights and the priors imposed on the images are incorporated into a variational framework to treat more complex mixed noise distribution. Such noise is characterized by heavy tails caused by impulse noise which needs to be eliminated through proper weighting of encoding residual. The outliers caused by the impulse noise has a significant effect on the encoding weights. Hence a more accurate residual encoding error initialization plays the important role in overall denoising performance, especially at high impulse noise rates. In this paper, outliers free initialization image, and an easier to implement a parameter-free procedure for updating encoding weights have been proposed. Experimental results demonstrate the capability of the proposed strategy to recover images highly corrupted by mixed Gaussian plus impulse noise as compared with the state of art denoising algorithm. The achieved results motivate us to implement the proposed algorithm in practice.
\end{abstract}

This is an open access article under the CC BY-SA license.

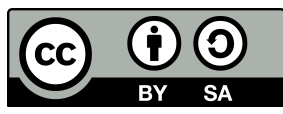

\section{Corresponding Author:}

Ahmed Abdulqader Hussein

Department of Electromechanical Engineering

University of Technology, Baghdad, Iraq

Email: ahmedabdulqaderhussein@gmail.com

\section{INTRODUCTION}

In various image applications, an image is inevitably polluted by noise of different types. Mixed noise could occur when an image that has already been contaminated by Gaussian noise in the procedure of image acquisition with faulty equipment suffers impulsive corruption during its transmission over noisy channels successively [1]. The main objective of any denoising algorithm is to remove noise as totally as possible and to preserve image edges as completely as possible. In a view of relevant studies, several denoising algorithms have been implemented for denoising a corrupted images by either impulse noise or Gaussian noise [2]-[6]. While, few relevant works have been proposed in order to remove mixed noise efficaciously due to the distinct characteristics of both kinds of degradation processes. Generally, enhanced Gaussian denoising algorithms are considered to be insufficient for suppressing the impulse noise due to the noisy pixels are translated as an edges and should be preserved. Meanwhile, the strategies for impulse noise elimination detect the impulse pixels and replace them with estimated values while leaving the remaining pixels unchanged. Along these lines, most Gaussian noise in the restored images will be kept leading to grainy and unsatisfactory visual results. Thus, a specific algorithm needs to be designed for standing against mixed Gaussian plus impulse noise [7], [8].

The sparse representation (SR) has proved its effectiveness in dealing with various image processing 
fields [9]-[11]. The algorithms based on SR, benefit from the sparse nature of the images which can be well restricted by a linear combination of only few atoms of transformed domain called dictionary. The main weakness of SR model based denoising algorithms is its incapability in dealing with mixed noise due to complicated properties of mixed noise distribution. To overcome SR model limitations, a dedicated algorithm need to be carefully designed to deal with heavy tails of mixed noise caused by impulse noise. Integrating the SR into total variation framework with proper encoding residual weighting, open the door for appearing algorithms capable of eliminating mixed noise efficiently. A weighted encoding with sparse nonlocal regularization model named (WESNR) was successfully applied in [9].

The main goal of this paper is to enhance the performance of WESNR algorithm firstly by initializing the algorithm with a more accurate image to ensure proper residual encoding error initialization and secondly by suggesting simpler weight updating procedure that ensures proper weighting of encoding residual to guarantee cancelling the heavy tails of mixed noise distribution induced by impulse noise. The achieved results show that the proposed algorithm exhibits improved performance in dealing with images highly corrupted by mixed Gaussian plus impulse noise as well as facilitating the practical implementation conditions.

The rest of the paper is organized as follows. Related work is introduced in Section 2. Section 3 describes the mixed Gaussian plus impulse noise model used in this paper. Section 4 briefly reviews the core of sparse denoising model. The proposed image denoising algorithm is explained In Section 5. In section 6, the results of the proposed denoising algorithm is compared with WESNR [9]. Finally, the concluding remarks are given in section 7 .

\section{RELATED WORK}

A general adaptive framework for detection and elimination different types of noise, involving Gaussian noise, impulse noise and more importantly their mixtures has been proposed in [12]. The method is implemented based on maximum likelihood estimation (MLE) approach as well as sparse representations over a trained dictionary. A patch-based approach for removing mixed noise based on sparse representation has been presented in [13]. An optimization problem is formulated based on $\ell_{1}$-norm and $\ell_{0}$-quasiy-norm penalties. The sparse representation in a dictionary and sparsity of residual is enforced by $\ell_{0}-\ell_{1}$ penalties respectively. The dictionary is learned using independent component analysis (ICA). The image is denoised iteratively using a combination of soft and hard thresholding. Results show that the proposed method gave good results in terms of SSIM quantitative measure for images rich with fine details. However, the performance of the proposed algorithm is not verified under heavily mixed noise degradation. A weighted encoding with sparse nonlocal regularization (WESNR) that can deal with images corrupted by mixed noise has been implemented in [9]. Weighted encoding is utilized in order to handle the impulse and Gaussian noise jointly. The prior image sparsity as well as prior nonlocal self-similarity have been consolidated by means of a regularization term and inserted into the variational encoding mechanism. The major drawbacks of this algorithm is its incapability of dealing with highly corrupted images.

A nonparametric Bayesian model to solve sparse outlier has been proposed in [14], [15]. This strategy proves its effectiveness in treating mixed Gaussian plus impulse (salt and pepper) noise. The noisy image is considered to be composed of three terms namely clean image, Gaussian noise image, and sparse impulse noise image. The model employs spike-slob sparse prior to recognize sparse coefficients of the real data term and outlier noise. An algorithm for jointly denoising hyperspectral images corrupted by mixed Gaussian plus impulse noise has ben proposed in [16]-[18]. The proposed algorithm avails the inherent spatial and spectral correlation of such images. The denoising problem is formulated based on synthesis prior (SP) and solved using Split-Bregman algorithm.

A new statistical regularization term called joint adaptive statistical prior (JASP) has been presented in [19]. The proposed strategy is incorporated into variational scheme. Moreover, the proposed variational model is solved using Split-Bregman iterative algorithm to recover images corrupted by mixed Gaussian plus impulse noise. A weighted joint sparse representation model called WJSR to recover images corrupted by mixed noise has been presented in [10]. The model group similar image patches and solved for global optimal solution using weighted simultaneous orthogonal matching pursuit (W-SOMP). The weights are included to stands against impulse noise which abolishes image patch similarities. The WJSR is integrated, with the global and sparse error priors, into variational framework to efficiently deal with mixed noise corruption. The major drawback of this algorithm is its computation complexity due to the usage of greedy algorithm W-SOMP. 
An iterative non-convex low rank matrix approximation (NonLRMA) model for denoising hyperspectral image (HIS) corrupted by mixed noise has been proposed in [20]-[22]. NonLRMA decomposes the degraded HSI image into a low rank component and a sparse term. Results prove the capability of the proposed algorithm to preserve large-scale image structures and small-scale details over a wide range of image bands degradations. An adaptive Median based Non-local Low Rank Approximation (AMNLRA) approach for denoising images polluted by mixed gaussian plus impulse noise has been proposed in [23]. The proposed method is based on eliminating the effect of impulsive noise on the mixed noise probability distribution to recover the Gaussian probability distribution. To locate the position of pixels contaminated by impulse noise, an adaptive median filter AMF [24] was used. According to data redundancy, these identified pixels are then processed using non-local mean filtering (NLM) to convert the residual noise into a Gaussian noise distribution. Finally, the pre-processed image has been processed using reduced rank optimization to obtain the denoised image.

\section{PROBLEM FORMULATION}

In a view of image denoising process, the elimination of mixed noise is more complicated than the standardized single noise. As a rule, this noise is sampled from various distributions. In this paper, the proposed strategy gives more attention for image denoising of mixed noise which specified by a combination of Gaussian and impulse noise. Obviously, it is a considerable problem that can be modeled as (1):

$$
y=I M P(x+n)
$$

where, $x, y$ and $n$ denotes the original image pixel, corrupted image pixel and additive white Gaussian noise respectively. The symbol $I M P$, denotes the corruption process by salt and pepper impulse noise.

\section{SPARSE DENOISING MODEL}

Let us denote an image by $x \in R^{N}$. The extracted image patch, can be represented as (2):

$$
x_{j}=R_{j} x
$$

where $x_{j}$ the stretched vector of an image is a patch of size $\sqrt{n} \times \sqrt{n}$ and $R_{j}$ is the matrix extraction operator at location $j$. The goal of sparse representation theory is to find an over complete dictionary $D=$ $\left[d_{1}, d_{2}, \ldots \ldots . d_{n}\right] \in R^{n \times m}$ to sparsely code $x_{j}$ where $d_{j} \in R^{n}$ is the $j^{\text {th }}$ atom of $D$. Based on the derived dictionary $D$, the image patch can be rewritten according to (3) [9]:

$$
x_{j}=D \alpha_{j}
$$

where $\alpha_{j}$ is a sparse coding vector. The least square solution of $x$ can be expressed as (4):

$$
x=D \alpha
$$

where $\alpha$ is the set of all coding vectors $\alpha_{j}$. In case of AWGN corrupted image $y, \alpha$ can be expressed as (5) [9]:

$$
\hat{\alpha}=\arg _{\min \alpha}\|y-D \alpha\|_{2}^{2}+\lambda R(\alpha)
$$

where $R(\alpha)$ and $\lambda$ are the regularization term and regularization parameter that ensure maximum a posteriori probability (MAP) solution of $\alpha$ for AWGN model. However, for mixed noise removal, MAP solution is unreachable due to heavy tail caused by impulse noise. To deal with mixed noise removal, a new model named weighted encoding with sparse nonlocal regularization (WESNR) has been proposed. According to WESNR, The (5) is rewritten as [9]:

$$
\hat{\alpha}=\arg _{\min \alpha}\left\|W^{\frac{1}{2}}(y-D \alpha)\right\|_{2}^{2}+\lambda R(\alpha)
$$

where $W$ is a diagonal weights matrix its elements $W_{j j}$ is derived from residual error $e_{j}=(y-D \alpha)_{j}$ as [9]:

$$
W_{j j}=\exp \left(-c e_{j}^{2}\right)
$$


where $c$ is a positive constant to control the decreasing rate of $W_{j j}$. The weights $W$ are adaptively updated in each iteration. The pixel corrupted by impulse noise is assigned small weight close to zero, while the uncorrupted pixel is left unchanged through assigning weight close to 1 . This process will eliminate the effect of heavy tails caused by impulse noise and hence ensure MAP solution of (6) to recover images corrupted by mixed Gaussian plus impulse noise.

The updating of $W$ depends on the coding residual error $e$. In [9], the initialized image $x(0)$ for salt and pepper impulse noise is obtained by applying AMF [24] to $y$. Thus, the residual error is initialized according to (8).

$$
e(0)=y-x(0)
$$

Inspired by the work in [25], local sparsity and nonlocal self-similarity are the two priors adopted in WESNR to model regularization term $R(\alpha)$ yields to (9):

$$
\hat{\alpha}=\arg _{\min \alpha}\left\|W^{\frac{1}{2}}(y-D \alpha)\right\|_{2}^{2}+\lambda\|\alpha-\mu\|_{1}
$$

as proposed in [9], the (9) was solved via iteratively reweighted least square minimization scheme which has been proposed in [26] using the (10):

$$
v_{j j}(k+1)=\frac{\lambda}{\sqrt{\left(\alpha_{j}(k)-\mu_{j}\right)^{2}+\varepsilon(k)^{2}}}
$$

where $\alpha_{j}(k)$ and $\varepsilon(k)$ is the $j^{t h}$ element of coding vector $\alpha$ and numerical stability parameter in the $k^{t h}$ iteration respectively. Hence, $\varepsilon(k)$ as well as $\alpha$ are updated according to (11) and (12) respectively [9].

$$
\begin{gathered}
\varepsilon(k+1)=\min (\varepsilon(k), \text { median }(|\alpha(k)-\mu|)) \\
\alpha(k+1)=\left(D^{T} W D+V(k+1)\right)^{-1}\left(D^{T} W y-D^{T} W D \mu\right)+\mu
\end{gathered}
$$

\section{THE PROPOSED ALGORITHM}

In WESNR [9], the weights $W$ have the important role in attenuating the effect of heavy tails triggered by impulse noise and hence retrieve back the distribution of mixed Gaussian plus impulse noise to resemble Gaussian noise distribution. This will pave the way for sparse modeling technique to implement denoising algorithms that can deal with images corrupted by mixed noise without explicit impulse detection phase. Referring to (6), the accuracy of estimated sparse vector $\hat{\alpha}$ and the diagonal weight matrix $W$ are highly affected by residual encoding error which in turn depends upon the initialized image $x(0)$. Thus, better performance is expected when more accurate image is used for initialization. Generally, the salt and pepper impulse noise take either minimum or maximum values in the dynamic range of the image (e.g. $[0,255])$. Thus, we need to process the pixels that take these extreme values. The question raised now is how to replace the corrupted pixel in such a way that ensures resemblance with its noise free neighbor pixels and hence dimensions the effect of outliers. Intuitively, critical replacements occurs when the test window $\Omega_{u, z}$ centered at pixel $p_{u, z}$ filled with impulses. To solve this problem, we suggest to adopt the Robust Outlier Exclusion (ROE) proposed in [27]. In this work the test window size is expanded to the next higher one (typically $4 \times 4$ ) and apply the trimmed mean which is characterized by its robustness against outliers. The conception for limiting the maximum test window size to $4 \times 4$ is to boost the estimated accuracy without influencing the significant correlation characteristics of neighbor pixels. As stated in [27], 25\% of the outliers exclusion accomplishes a powerful estimatation for recovering the corrupted pixels. Algorithm 1 shows the initialization algorithm steps according to ROE [27].

Image mixed gaussian and impulse noise elimination based on sparse ... (Ahmed Abdulqader Hussein) 


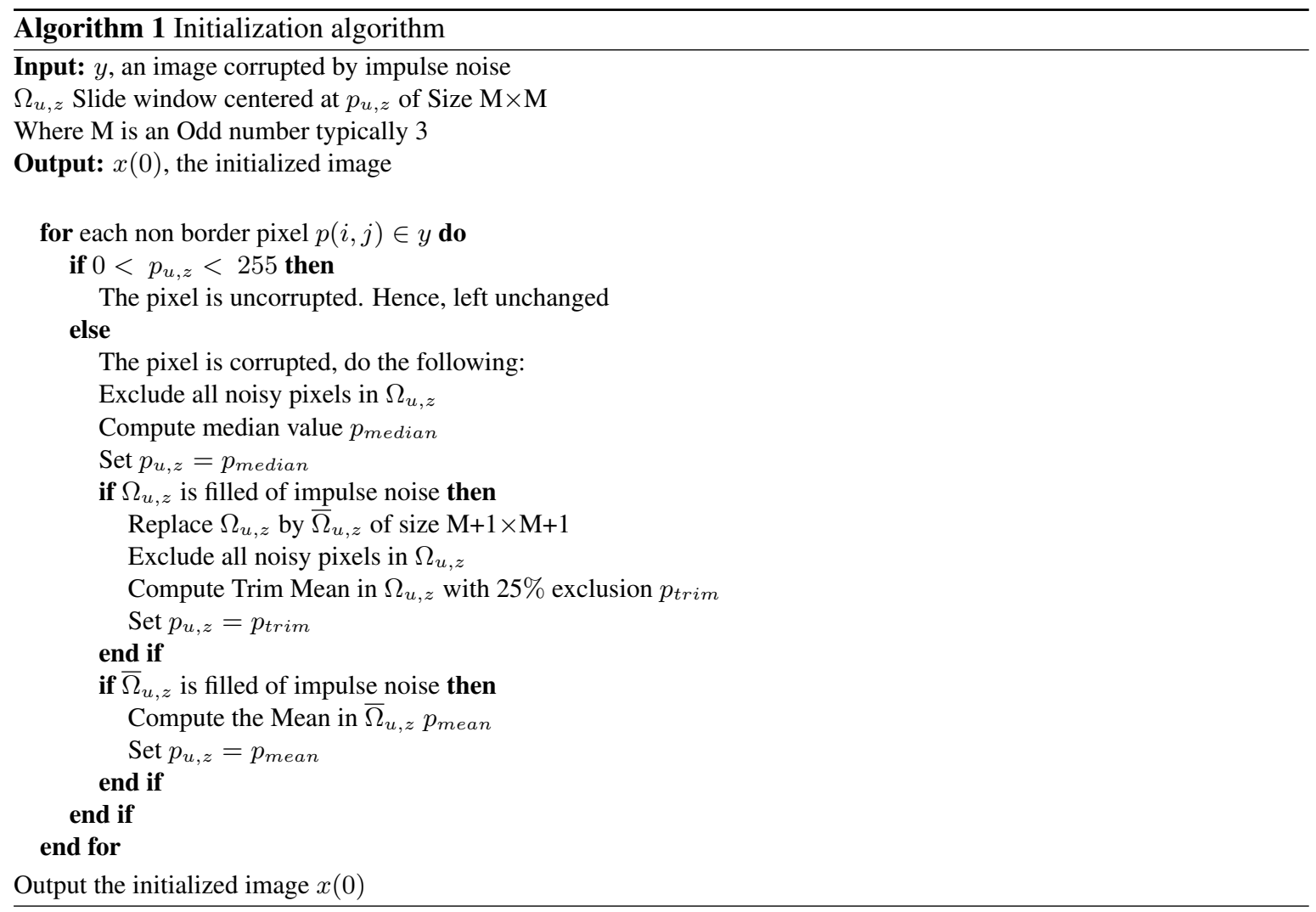

The initialization image $x(0)$ is now used to calculate the residual encoding error which plays important role in updating the weight matrix $W$. Referring to (7), the assigned weights $W$ is firstly sensitive to the value of the constant $c$ which controlling the decreasing rate of the weights and secondly use trigonometric exponential function. To modify the algorithm to suit the implementation requirements, it is usually best to avoid the use of mathematical functions, in this case, exponential functions. Hence, our goal is to replace the exponential function while retaining the properties of its execution. Referring to (7), impulse noise will cause a significant increase in residual error, which will lead to the assignation of weight close to zero $(W \rightarrow 0)$, thereby eliminating the impact of heavy tails caused by impulse noise. Otherwise, if the pixel has no impulse noise, the weight will be close to one $(W \rightarrow 1)$.

Now to achieve our goal, assuming $a$ is a positive value greater than zero and less or equal to 1 , then $(1-a)^{2} \rightarrow 0$ when $a \rightarrow 1$ and $(1-a)^{2} \rightarrow 1$ when $a \rightarrow 0$. The function $(1-a)^{2}$ has the same properties as the exponential function. Consequently, the diagonal weight matrix $W$ can be updated according to (13).

$$
W=(1-a)^{2} \quad 0 \leqslant a \leqslant 1
$$

The constraints imposed on the variable $a$ in (13) can be related to the absolute normalized residual coding error $\left(\left|e_{\text {norm }}\right|\right)$. Accordingly, the weight matrix $\mathrm{W}$ is updated through the following steps:

1. Calculate absolute residual error:

$$
e(k)=|(y-D \alpha(k))|
$$

2. Normalize the error by element wise division:

$$
e_{\text {norm }}(k)=\frac{e(k)}{\max (e(k))}
$$

3. Update weight diagonal matrix:

$$
W_{j j}(k)=\left(1-e_{n o r m}(k)\right)^{2}
$$


Regarding to the case of impulsive noise which is specified by (16), the normalized error $\left(\left|e_{\text {norm }}\right|\right)$ approaches 1 and thus a value close to zero will be assigned to the encoding weights $W_{j j}$ which eliminate the effect of the impulsive noise. Hence, ensuring MAP solution of (6) in order to recover images corrupted by mixed Gaussian plus impulse noise. Moreover, compared with (7) and (16) has the advantage of being easier to implement in practice by avoiding the use of an exponential function. Therefore, there is no need to calibrate the parameter $c$. By following the steps outlined above, Algorithm 2 demonstrates the modification of the proposed WESNR based mixed Gaussian plus impulse image denoising algorithm.

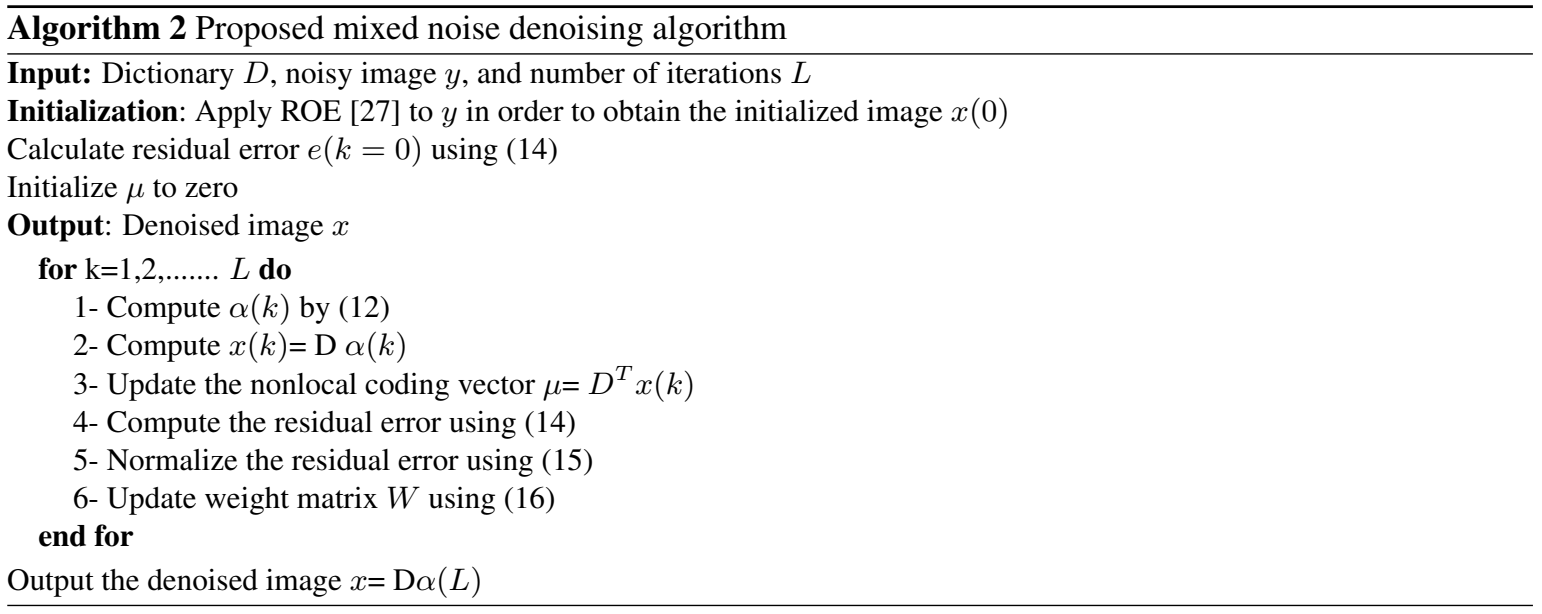

\section{EXPERIMENTAL RESULTS}

The proposed algorithm is evaluated on a set of standard images having distinctly different features of size $512 \times 512$. These images are artificially polluted firstly by Gaussian noise with standard deviation $\sigma$ of 10,20 , and 30 and then $30 \%, 50 \%$, and $70 \%$ of image pixels are replaced by salt and pepper impulsive noise. Objectively, the obtained results are evaluated using peak signal to noise ratio (PSNR) and the structural similarity index (SSIM) [28]. Moreover, subjective evaluation is included to prove the quality of the recovered images. The proposed algorithm is compared with WESNR [9]. The parameters of WESNR are set to its default values as proposed in [9]. The parameters are $c=0.0008, \lambda(0)=0.0001$ to reduce the effect of impulse noise on block-matching then $\lambda=1$ for $\sigma>10$ or $\lambda=0.5$ for $\sigma \leqslant 10, \varepsilon(0)=0.1$ and updated in accordance with (11). The image patches are encoded over a set of offline learned PCA based dictionaries. The proposed algorithm has the same parameters as WESNR except of the parameter $c$ which has been discarded. The number of iterations $L$ is kept fixed at 8 for all experiments.

\subsection{Objective evaluation}

The denoising relative performance in terms of PSNR and SSIM for an average of five runs using a set of images are recorded in Table 2 (the value in parenthesis is the SSIM). According to the performance criteria, the superior outcomes are referred with a bold font. Regarding to the results examination of Table 1, the proposed algorithm outperforms WESNR algorithm most of the time in terms of individual, average PSNR as well as SSIM values over the whole scope of noise levels and images under test.

In addition, by carefully checking the obtained results in Table 2, it is noticed that as the level of mixed noise increases, the proposed algorithm exhibits higher performance compared to WESNR. As an illustration, Figure 1a, Figure 1b, Figure 2a, and Figure 2b demonstrate the relative denoising of both algorithms in terms of PSNR and SSIM for $\sigma=30, s p=30 \%$, and $\sigma=30, s p=70 \%$ respectively. The achieved results prove the enhanced performance and the effectiveness of the modification inserted on the conventional WESNR algorithm namely starting with a more accurate initialized image $x(0)$. The more accurate initialization of the proposed algorithm has been reflected in increasing the accuracy of estimating the initialized weight matrix $W$. Furthermore, the proposed function for updating the diagonal weight matrix $W$ in (16) proves its ability to attenuate the effect of heavy tails caused by impulse noise considerably. Therefore, a MAP solution for recovering images corrupted by mixed Gaussian plus impulse noise has been realized. 
Table 1. PSNR (SSIM) denoising results

\begin{tabular}{|c|c|c|c|c|c|c|c|}
\hline \multirow{2}{*}{ Images } & \multirow{2}{*}{$\sigma$} & \multicolumn{2}{|c|}{ sp $30 \%$} & \multicolumn{2}{|c|}{ sp 50\% } & \multicolumn{2}{|c|}{ sp 70\% } \\
\hline & & WESNR & Proposed & WESNR & Proposed & WESNR & Proposed \\
\hline \multirow{6}{*}{ Lena } & & 33.31 & 33.84 & 31.79 & 32.50 & 29.31 & 29.85 \\
\hline & 10 & $(0.879)$ & $(0.881)$ & $(0.863)$ & $(0.867)$ & $(0.830)$ & $(0.835)$ \\
\hline & & 30.68 & 31.03 & 29.61 & 30.19 & 27.59 & 28.17 \\
\hline & 20 & $(0.810)$ & $0.818)$ & $(0.796)$ & $(0.807)$ & $(0.769)$ & $(0.787)$ \\
\hline & & 27.23 & 27.97 & 26.00 & 27.94 & 24.01 & 26.69 \\
\hline & 30 & $(0.634)$ & (0.657) & $(0.603)$ & $(0.633)$ & $(0.577)$ & $(0.620)$ \\
\hline \multirow{6}{*}{ Boat } & & 30.56 & 31.39 & 28.73 & 29.56 & 26.12 & 26.87 \\
\hline & 10 & $(0.831)$ & $(0.845)$ & $(0.799)$ & $(0.808)$ & $(0.736)$ & $(0.739)$ \\
\hline & & 28.27 & 28.68 & 26.89 & 27.54 & 24.86 & 25.49 \\
\hline & 20 & $(0.747)$ & $(0.751)$ & $(0.719)$ & $(0.721)$ & $(0.657)$ & $(0.667)$ \\
\hline & & 25.83 & 26.39 & 24.34 & 25.93 & 22.29 & 24.45 \\
\hline & 30 & $(0.619)$ & (0.642) & $(0.582)$ & (0.635) & $(0.532)$ & $(0.612)$ \\
\hline \multirow{6}{*}{ Hill } & & 30.88 & 31.45 & 29.77 & 30.11 & 27.98 & 28.13 \\
\hline & 10 & $(0.815)$ & $(0.830)$ & $(0.785)$ & (0.791) & $(0.724)$ & $0.728)$ \\
\hline & & 28.74 & 29.01 & 27.86 & 28.11 & 26.39 & 26.57 \\
\hline & 20 & $(0.725)$ & $(0.731)$ & (0.699) & $(0.704)$ & $(0.652)$ & $(0.654)$ \\
\hline & & 26.27 & 26.81 & 25.22 & 26.54 & 23.60 & 25.62 \\
\hline & 30 & (0.599) & (0.622) & $(0.558)$ & (0.618) & $(0.516)$ & (0.604) \\
\hline \multirow{6}{*}{ F16 } & & 32.47 & 29.46 & 30.61 & 28.84 & 27.72 & 27.20 \\
\hline & 10 & $(0.893)$ & $(0.889)$ & $(0.878)$ & $(0.878)$ & $(0.846)$ & $(0.851)$ \\
\hline & & 30.14 & 28.18 & 28.72 & 27.68 & 26.32 & 26.21 \\
\hline & 20 & $(0.824)$ & $(0.816)$ & $(0.813)$ & (0.819) & $(0.787)$ & $(0.806)$ \\
\hline & & 26.79 & 26.32 & 25.65 & 26.20 & 23.28 & 25.22 \\
\hline & 30 & $(0.627)$ & (0.653) & $(0.607)$ & (0.635) & $(0.564)$ & $(0.602)$ \\
\hline \multirow{6}{*}{ Monarch } & & 35.36 & 35.78 & 33.09 & 34.29 & 28.72 & 29.83 \\
\hline & 10 & (0.935) & (0.937) & $(0.930)$ & (0.933) & (0.900) & $(0.913)$ \\
\hline & & 31.66 & 32.04 & 30.03 & 31.16 & 26.68 & 28.10 \\
\hline & 20 & $(0.860)$ & (0.880) & $(0.861)$ & $(0.870)$ & $(0.830)$ & (0.849) \\
\hline & & 27.51 & 28.31 & 25.92 & 28.24 & 23.00 & 26.15 \\
\hline & 30 & (0.674) & $(0.804)$ & $(0.644)$ & (0.787) & $(0.600)$ & $(0.754)$ \\
\hline \multirow{6}{*}{ Barbara } & & 29.18 & 31.44 & 26.99 & 28.82 & 23.97 & 25.21 \\
\hline & 10 & (0.874) & (0.899) & $(0.831)$ & $(0.860)$ & $(0.728)$ & (0.759) \\
\hline & & 27.13 & 28.65 & 25.44 & 26.65 & 22.75 & 23.88 \\
\hline & 20 & $(0.793)$ & $(0.822)$ & $(0.744)$ & $(0.773)$ & $(0.651)$ & (0.677) \\
\hline & & 24.57 & 25.96 & 22.64 & 25.02 & 20.49 & 23.20 \\
\hline & 30 & (0.644) & (0.692) & $(0.587)$ & (0.681) & $(0.505)$ & $(0.624)$ \\
\hline & & 36.97 & 36.87 & 36.02 & 36.21 & 33.51 & 33.62 \\
\hline House & 10 & $(0.912)$ & $(0.902)$ & $(0.904)$ & $(0.900)$ & $(0.886)$ & $(0.890)$ \\
\hline & & 33.67 & 33.76 & 32.78 & 33.48 & 30.84 & 31.45 \\
\hline & 20 & $(0.843)$ & $(0.862)$ & $(0.837)$ & (0.859) & $(0.825)$ & $(0.848)$ \\
\hline & & 28.90 & 29.70 & 27.83 & 29.04 & 25.83 & 28.31 \\
\hline & 30 & $(0.630)$ & (0.777) & $(0.610)$ & $(0.713)$ & (0.598) & $(0.654)$ \\
\hline & & 30.34 & 31.17 & 28.77 & 29.50 & 26.18 & 26.77 \\
\hline Couple & 10 & $(0.846)$ & $(0.860)$ & $(0.813)$ & $(0.824)$ & $(0.738)$ & $(0.744)$ \\
\hline & 20 & 28.04 & 28.52 & 26.84 & 27.32 & 24.77 & 25.20 \\
\hline & 20 & $(0.757)$ & $(0.765)$ & $(0.725)$ & $(0.728)$ & $(0.641)$ & $(0.654)$ \\
\hline & & 25.56 & 26.12 & 24.37 & 25.63 & 22.51 & 24.19 \\
\hline & 30 & $(0.626)$ & (0.643) & $(0.592)$ & (0.637) & $(0.536)$ & (0.596) \\
\hline Man & 10 & 30.71 & 31.18 & 29.36 & 29.80 & 27.18 & 27.61 \\
\hline Man & 10 & $(0.841)$ & (0.849) & $(0.814)$ & $(0.818)$ & $(0.758)$ & $(0.761)$ \\
\hline & & 28.51 & 28.74 & 27.65 & 27.91 & 25.69 & 26.14 \\
\hline & 20 & $(0.753)$ & $(0.755)$ & $(0.734)$ & $(0.737)$ & $(0.679)$ & $(0.690)$ \\
\hline & & 26.09 & 26.60 & 24.86 & 26.25 & 22.97 & 25.23 \\
\hline & 30 & $(0.613)$ & $(0.653)$ & $(0.577)$ & (0.644) & $(0.529)$ & $(0.636)$ \\
\hline & & 34.73 & 34.99 & 33.83 & 34.06 & 32.10 & 32.23 \\
\hline Zelda & 10 & (0.884) & $(0.886)$ & $(0.872)$ & $(0.875)$ & $(0.847)$ & $(0.855)$ \\
\hline & & 31.87 & 32.14 & 31.05 & 31.53 & 29.52 & 30.09 \\
\hline & 20 & $(0.815)$ & $(0.818)$ & $(0.802)$ & $(0.815)$ & (0.779) & (0.807) \\
\hline & & 28.10 & 29.76 & 27.14 & 28.48 & 25.60 & 27.71 \\
\hline & 30 & $(0.645)$ & $(0.747)$ & $(0.630)$ & $(0.717)$ & $(0.613)$ & $(0.705)$ \\
\hline & & 32.45 & 32.76 & 30.90 & 31.37 & 28.28 & 28.73 \\
\hline Average & 10 & $(0.871)$ & (0.878) & (0.849) & $(0.855)$ & $(0.800)$ & $(0.808)$ \\
\hline & & 29.87 & 30.08 & 28.69 & 29.16 & 26.54 & 27.13 \\
\hline & 20 & (0.793) & $(0.802)$ & $(0.773)$ & $(0.783)$ & $(0.727)$ & (0.744) \\
\hline & & 26.69 & 27.39 & 25.40 & 26.93 & 23.36 & 25.68 \\
\hline & 30 & $(0.631)$ & $(0.690)$ & $(0.600)$ & $(0.670)$ & $(0.557)$ & $(0.641)$ \\
\hline
\end{tabular}

Indonesian J Elec Eng \& Comp Sci, Vol. 23, No. 3, September 2021 : 1440 - 1450 
In order to complete the picture as a whole, Figures 3 and Figures 4 show the relative average PSNR and SSIM performance for all images under the influence of different mixing noise levels. In view of the accomplished results of Figures 3 and 4. the proposed algorithm performance has been differentiated at $\sigma=$ 30 compared to the WESNR algorithm. Along these lines, more accurate processing of impulse noise through ROE [27] has the greatest impact on the quality of denoised images, especially when $s p$ exceeds $50 \%$.

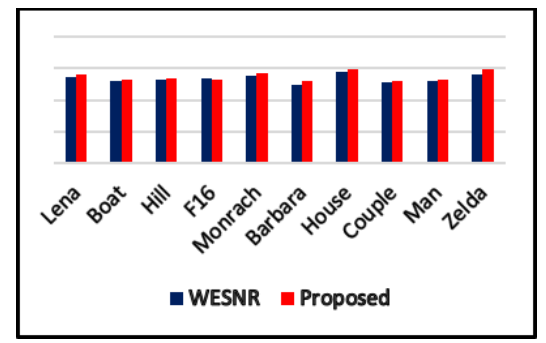

(a)

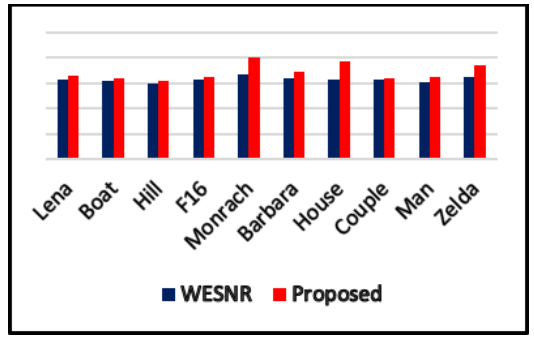

(b)

Figure 1. Relative denoising algorithms performance for $\sigma=30, s p=30 \%$; (a) PSNR (dB) and (b) SSIM

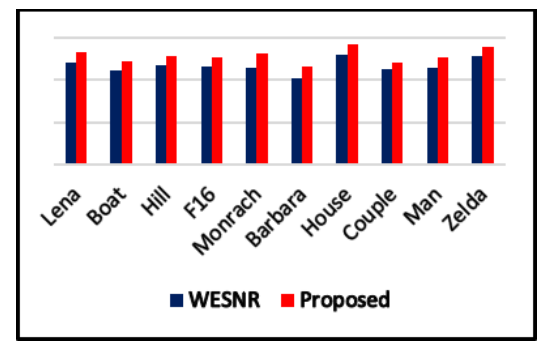

(a)

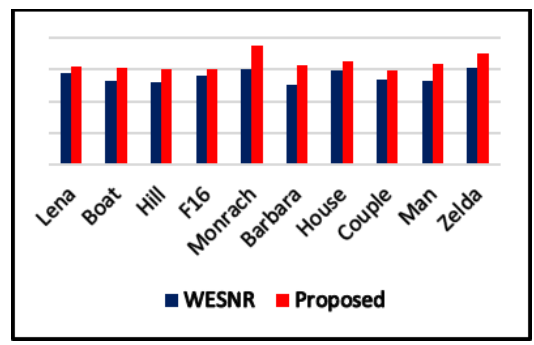

(b)

Figure 2. Relative denoising algorithms performance for $\sigma=30, s p=70 \%$; (a) PSNR (dB) and (b) SSIM

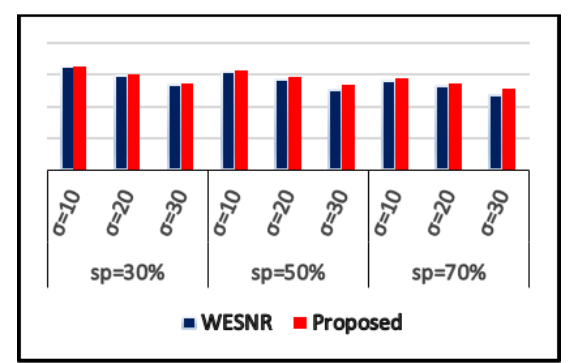

(a)

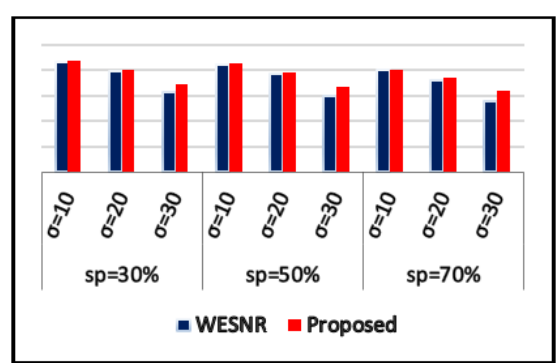

(b)

Figure 3. Relative average denoising performance for different mixed noise levels;

(a) PSNR (dB) and (b) SSIM

Finally, in order to quantitatively summarize the results in the case of high mixed noise levels of $\sigma=30$, $s p=70 \%$, the proposed algorithm achieves an average PSNR gain of approximately $2.32 \mathrm{~dB}$ over the entire set of images under severe noise conditions. 


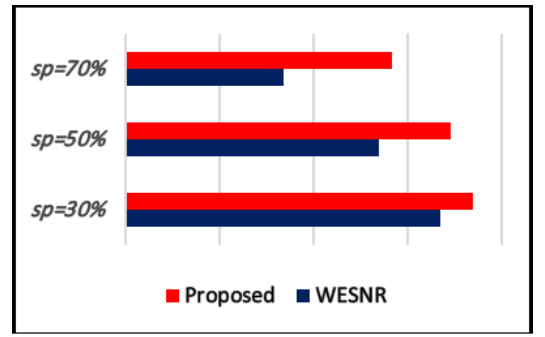

(a)

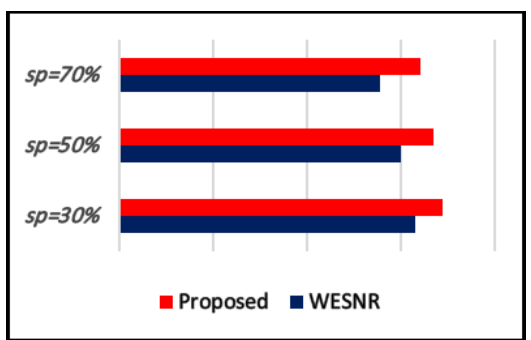

(b)

Figure 4. Relative average denoising algorithms performance for $\sigma=30$; (a) PSNR (dB) and (b) SSIM

\subsection{Subjective evaluation}

In accordance to the performance analysis of the proposed approach, it is significant to integrate the visual comprehension in the relative performance comparison. Figure 5 illustrates a sample of images under test synthetically corrupted by different levels of mixed Gaussian plus impulse noise. The first column of Figure 5 shows the original image of Monarch, Barbara, and house respectively. The subsequent columns demonstrate the noisy and the denoised images respectively. Clearly, for $\sigma=10$ and $s p=30 \%$, both algorithms achieve nearly equivalent performance (see Figure 5(a1-a4). The performance of the WESNR is degraded as the mixed noise level increased. The degradations become more obvious when $\sigma$ exceeds 20 and $s p$ exceeds $50 \%$. At a such high mixed noise environment, the proposed algorithm produces visually more pleasant results which can be easily noticed through Figure 5(b1-b4) and Figure 5(c1-c4). The proposed algorithm preserves the texture of the image in Figure 5(b4) and smooths the sky in Figure 5(c4) more accurately as compared with WESNR. These results demonstrate the effectiveness of the proposed procedure for updating the weights and to the replacement of AMF [24] adopted in WESNR by ROE [27] which in combination reduces the effect of heavy tails of the impulse noise, especially at high impulse noise density rates.

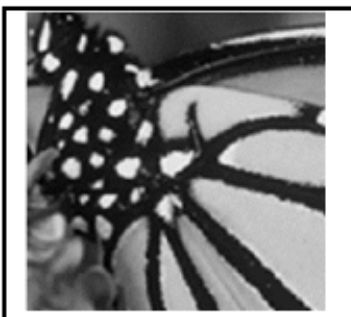

(a1) Monarch image

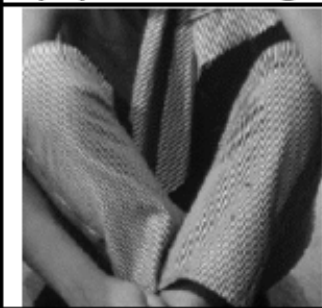

(b1) Barbara image

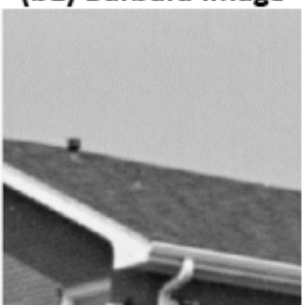

(c1) House image

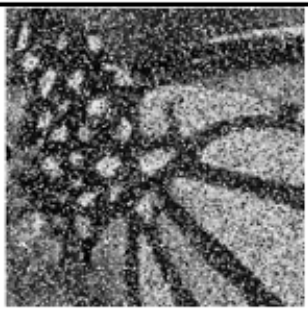

(a2) $\sigma=10, s p=30 \%$

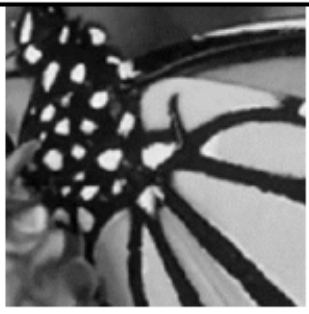

(a3) WESNR

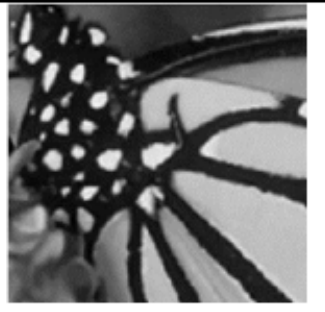

(a4) Proposed
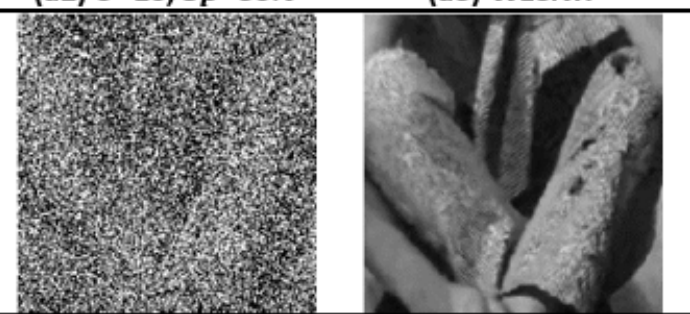

(b3) WESNR

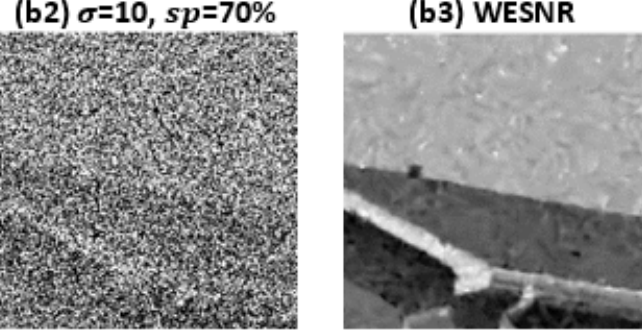

(c3) WESNR

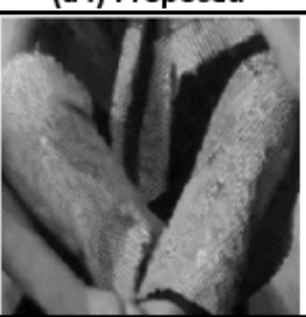

(b4) Proposed

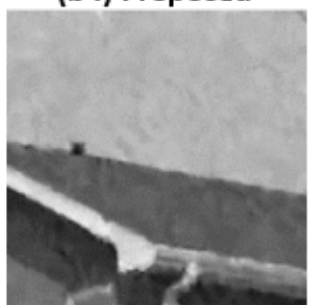

(c4) Proposed

Figure 5. Denoising results of mixed gaussian plus impulse noise 


\section{CONCLUSION}

This paper proposes a denoising algorithm in order to recover images highly corrupted by mixed Gaussian plus impulse noise. The promising results show that the proposed algorithm preserves image fine details more accurately as compared with WESNR especially for highly corrupted images. The main contributions of the proposed algorithm are the employment of a more accurate initialization image through replacing AMF by ROE and the use of a simple parameter-free procedure for updating encoding weights $W$ by avoiding the use of the exponential function and the need for calibrating the decaying rate parameter $(c)$ in accordance with the noise state level. As a quantitative example of the improvement in results, for $\sigma=30$ and $s p=70 \%$, the proposed algorithm accomplishes an average PSNR gain of approximately $2.32 \mathrm{~dB}$ over the entire images under test.The promising results of the proposed algorithm open the way for further improvement, enabling it to eliminate more complex Gaussian plus random valued impulse noise. This issue is left as a possible future work.

\section{REFERENCES}

[1] Y.-B. Zheng, T.-Z. Huang, X.-L. Zhao, T.-X. Jiang, T.-H. Ma, and T.-Y. Ji, "Mixed noise removal in hyperspectral image via low-fibered-rank regularization," IEEE Transactions on Geoscience and Remote Sensing, vol. 58, no. 1, pp. 734-749, Jan. 2020, doi: 10.1109/TGRS.2019.2940534.

[2] Y. Chen, J. Li, and Y. Zhou, "Hyperspectral image denoising by total variation-regularized bilinear factorization," Signal Processing, vol. 174, p. 107645, 2020, doi: 10.1016/j.sigpro.2020.107645.

[3] J. Chen, G. Zhang, S. Xu, and H. Yu, "A blind cnn denoising model for random-valued impulse noise," IEEE Access, vol. 7, pp. 124647-124661, 2019, doi: 10.1109/ACCESS.2019.2938799.

[4] H. Zeng, X. Xie, W. Kong, S. Cui, and J. Ning, "Hyperspectral image denoising via combined non-local selfsimilarity and local low-rank regularization," IEEE Access, vol. 8, pp. 50190-50208, 2020, doi: 10.1109/ACCESS.2020.2979809.

[5] S. A. Hussain, A. A. Hussein, and A. H. Reja, "Fast and robust random-valued image denoising algorithm based on road statistics," Proceedings of 187th The IIER International Conference, Hong Kong, 2018, pp. 12-18.

[6] A. Awad, "Removal of fixed-valued impulse noise based on probability of existence of the image pixel," International Journal of Electrical \& Computer Engineering (IJECE), vol. 8, no. 4, 2018, pp. 2106 2114, doi: 10.11591/ijece.v8i4.pp2106-2114.

[7] H. Zhu and M. K. Ng, "Structured dictionary learning for image denoising under mixed gaussian and impulse noise," IEEE Transactions on Image Processing, vol. 29, pp. 6680-6693, 2020, doi: 10.1109/TIP.2020.2992895.

[8] V. S. Tallapragada, N. A. Manga, G. P. Kumar, and M. V. Naresh, "Mixed image denoising using weighted coding and non-local similarity," SN Applied Sciences, vol. 2, no. 6, pp. 1-11, 2020, doi: 10.1007/s42452-020-2816-y.

[9] J. Jiang, L. Zhang, and J. Yang, "Mixed noise removal by weighted encoding with sparse nonlocal regularization," IEEE transactions on image processing, vol. 23, no. 6, pp. 2651-2662, June 2014, doi: 10.1109/TIP.2014.2317985.

[10] L. Liu, L. Chen, C. L. P. Chen, Y. Y. Tang and C. M. pun, "Weighted joint sparse representation for removing mixed noise in image," IEEE transactions on cybernetics, vol. 47, no. 3, pp. 600-611, March 2017, doi: 10.1109/TCYB.2016.2521428.

[11] L. Liu, C. P. Chen, X. You, Y. Y. Tang, Y. Zhang, and S. Li, "Mixed noise removal via robust constrained sparse representation," IEEE Transactions on Circuits and Systems for Video Technology, vol. 28, no. 9, pp. 2177-2189, Sept. 2018, doi: 10.1109/TCSVT.2017.2722232.

[12] J. Liu, X.-C. Tai, H. Huang, and Z. Huan, "A weighted dictionary learning model for denoising images corrupted by mixed noise," IEEE transactions on image processing, vol. 22, no. 3, pp. 1108-1120, March 2013, doi: 10.1109/TIP.2012.2227766.

[13] M. Filipović and A. Jukić, "Restoration of images corrupted by mixed gaussian-impulse noise by iterative soft-hard thresholding," in 2014 22nd European Signal Processing Conference (EUSIPCO), 2014, pp. 1637-1641.

[14] P. Zhuang, W. Wang, D. Zeng, and X. Ding, "Robust mixed noise removal with non-parametric bayesian sparse outlier model," in 2014 IEEE 16th International Workshop on Multimedia Signal Processing (MMSP), 2014, pp. 1-5, doi: 10.1109/MMSP.2014.6958792.

[15] P. Zhuang, Y. Huang, D. Zeng, and X. Ding, "Mixed noise removal based on a novel non-parametric bayesian sparse outlier model," Neurocomputing, vol. 174, pp. 858-865, 2016, doi: 10.1016/j.neucom.2015.09.095.

[16] H. K. Aggarwal and A. Majumdar, "Mixed gaussian and impulse denoising of hyperspectral images," in 2015 IEEE International Geoscience and Remote Sensing Symposium (IGARSS), 2015, pp. 429-432, doi: 10.1109/IGARSS.2015.7325792.

[17] H. K. Aggarwal and A. Majumdar, "Hyperspectral image denoising using spatio-spectral total variation," IEEE Geoscience and Remote Sensing Letters, vol. 13, no. 3, pp. 442-446, March 2016, doi: 10.1109/LGRS.2016.2518218. 
[18] S. Takeyama, S. Ono, and I. Kumazawa, "Hyperspectral image restoration by hybrid spatio-spectral total variation," in 2017 IEEE International Conference on Acoustics, Speech and Signal Processing (ICASSP), 2017, pp. 4586-4590, doi: 10.1109/ICASSP.2017.7953025

[19] N. Eslahi, H. Mahdavinataj, and A. Aghagolzadeh, "Mixed gaussian-impulse noise removal from highly corrupted images via adaptive local and nonlocal statistical priors," in 2015 9th Iranian Conference on Machine Vision and Image Processing (MVIP), 2015, pp. 70-75, doi: 10.1109/IranianMVIP.2015.7397507.

[20] Y. Chen, Y. Guo, Y. Wang, D. Wang, C. Peng, and G. He, "Denoising of hyperspectral images using nonconvex low rank matrix approximation," IEEE Transactions on Geoscience and Remote Sensing, vol. 55, no. 9, pp. 5366-5380, Sept. 2017, doi: 10.1109/TGRS.2017.2706326.

[21] Q. Wang, Z. Wu, J. Jin, T. Wang, and Y. Shen, "Low rank constraint and spatial spectral total variation for hyperspectral image mixed denoising," Signal Processing, vol. 142, pp. 11-26, 2018, doi: 10.1016/j.sigpro.2017.06.012.

[22] W. He, H. Zhang, L. Zhang, and H. Shen, "Hyperspectral image denoising via noise-adjusted iterative low-rank matrix approximation," IEEE Journal of Selected Topics in Applied Earth Observations and Remote Sensing, vol. 8, no. 6, pp. 3050-3061, June 2015, doi: 10.1109/JSTARS.2015.2398433.

[23] D. G. Kim, M. Hussain, M. Adnan, M. A. Farooq, Z. H. Shamsi, and A. Mushtaq, "Mixed noise removal using adaptive median based non-local rank minimization," IEEE Access, vol. 9, pp. 6438-6452, 2021, doi: 10.1109/ACCESS.2020.3048181.

[24] H. Hwang and R. A. Haddad, "Adaptive median filters: new algorithms and results," IEEE Transactions on image processing, vol. 4, no. 4, pp. 499-502, April 1995, doi: 10.1109/83.370679.

[25] W. Dong, L. Zhang, G. Shi, and X. Li, "Nonlocally centralized sparse representation for image restoration," IEEE transactions on Image Processing, vol. 22, no. 4, pp. 1620-1630, April 2013, doi: 10.1109/TIP.2012.2235847.

[26] I. Daubechies, R. DeVore, M. Fornasier, and C. S. Güntürk, "Iteratively reweighted least squares minimization for sparse recovery," Communications on Pure and Applied Mathematics: A Journal Issued by the Courant Institute of Mathematical Sciences, vol. 63, no. 1, pp. 1-38, 2010, doi: 10.1002/cpa.20303.

[27] S. A. Hussain and S. M. Gorashi, "Robust outliers exclusion for high density impulse noise suppression algorithm," in 2013 IEEE International Conference on Signal and Image Processing Applications, 2013, pp. 43-48, doi: 10.1109/ICSIPA.2013.6707975.

[28] Z. Wang, A. C. Bovik, H. R. Sheikh, and E. P. Simoncelli, "Image quality assessment: from error visibility to structural similarity," IEEE transactions on image processing, vol. 13, no. 4, pp. 600-612, April 2004, doi: 10.1109/TIP.2003.819861. 